\title{
PREDICTIVE THERMAL MODEL FOR INDIRECT TEMPERATURE MEASUREMENT INSIDE ATOMIC CELL OF NUCLEAR MAGNETIC RESONANCE GYROSCOPE
}

\author{
M. Salleras ${ }^{*}$, E.J. Eklund, I.P. Prikhodko, and A.M. Shkel \\ Microsystems Laboratory, University of California, Irvine, California, USA
}

\begin{abstract}
This paper describes a methodology for predicting temperatures inside a micro-device at critical locations where thermal sensors cannot be placed. The device under study is a nuclear magnetic resonance gyroscope, an instrument that is highly sensitive to temperature variations. A physical thermal model of the device is developed and validated using experimental measurements from a prototype. A compact thermal model which approximates the behavior of the physical system is obtained through a sequential selection algorithm (with RMS error of approximately $0.15^{\circ} \mathrm{C}$ ). The compact thermal model can be solved rapidly and is therefore suitable for real-time feedback control of the temperature inside the atomic cell.
\end{abstract}

\section{KEYWORDS}

Predictive models, atomic gyroscope, nuclear magnetic resonance, atomic MEMS, compact thermal model.

\section{INTRODUCTION}

We present a two-step process for predicting temperatures inside a device at critical locations where high accuracy is required and direct temperature measurements are not feasible due to generation of parasitic magnetic fields by a temperature sensor, limited space inside the device, or inaccessibility of the area of interest. The methodology described in this paper can be applied to a broad class of atomic MEMS devices. Conventional approaches are effectively open-loop, and do not yield sufficient precision demanded by recent emerging atomic MEMS applications [1].

A Finite Element (FE) model of the device is directly correlated to experimental measurements by matching and using an optimization algorithm. We then develop a Compact Thermal Model (CTM) which reproduces the results of the FE model, with high accuracy, at the critical locations of interest inside the device, such as inside of the atomic cell.

The NMR gyroscope [2-3] is a micromachined sensor which measures inertial rotation by detecting the NMR frequency shift in hyperpolarized noble gases (Fig. 1). A circularly polarized laser beam travels through optically pumped alkali vapor and noble gases confined in a cell. The laser frequency is tuned to the resonant absorption frequency of the alkali isotope. The light transmitted through the cell is collected on a photodetector, and inertial rotation is measured by detecting a shift in the nuclear precession frequency, which is embedded in the optical signal due to spin-exchange between the nuclei and the alkali vapor [4]. The degree of polarization is highly dependent on the cell temperature, and temperature fluctuations in the cell can therefore create undesirable shifts in the signal. The temperature sensitivity of an NMR gyroscope is theoretically estimated to be on the order of $1 \mathrm{deg} / \mathrm{h} / \mathrm{mK}$ [5-6]. Therefore the temperature stability of the cell is required to be better than $1 \mathrm{mK}$ to achieve the targeted sub-degree per hour gyroscope performance.

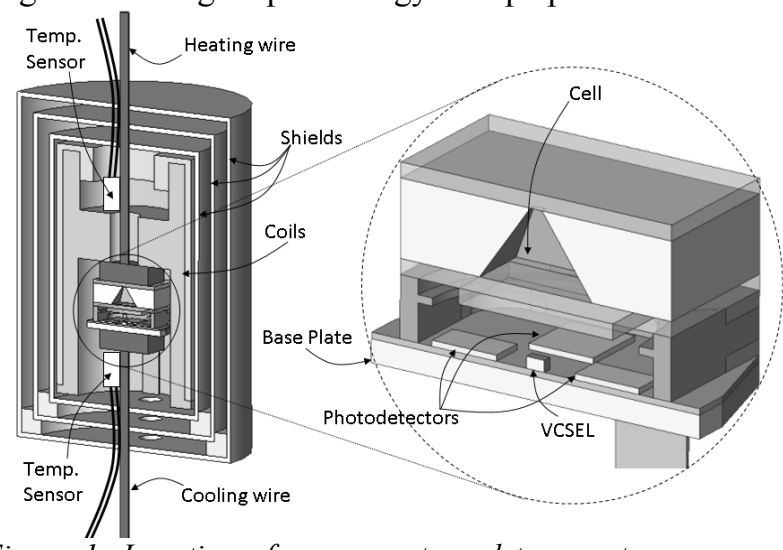

Figure 1: Location of components and temperature sensors in the NMR gyroscope.

High vapor pressure of the confined alkali is needed to achieve a sufficiently strong absorption signal. ${ }^{87} \mathrm{Rb}$ isotope is chosen for its high vapor pressure at relatively low temperatures. A cell temperature of $120^{\circ} \mathrm{C}$ is chosen to achieve high polarization with uniform distribution in the cell [6]. In addition, the light source, a Vertical-Cavity Surface-Emitting Laser (VCSEL), must operate at temperatures below $80^{\circ} \mathrm{C}$ to avoid degradation and reduced lifetime. Due to the close proximity of the cell and the VCSEL, active heating and cooling was included in the presented prototype through an external heater and a ThermoElectric Cooler (TEC) (Fig. 1). The cell and VCSEL temperatures need to be measured and controlled to ensure that they satisfy the stringent thermal requirements.

\section{EXPERIMENTAL MEASUREMENTS}

An NMR gyroscope prototype was built, Fig. 1, and thermal experiments conducted on the setup shown in Fig. 2 . The NMR gyroscope prototype and signal conditioning electronics were enclosed in a metallic box to reduce noise in the measurements. A DAQ board from National Instruments was used to acquire the gyroscope signal and 
temperature measurements. Heater and TEC controllers (TC200 and TED200C from Thorlabs) were used to tune the heating and cooling temperatures. Function generators were used to sweep the VCSEL current and to supply the signals for magnetic field generation in the gyroscope.

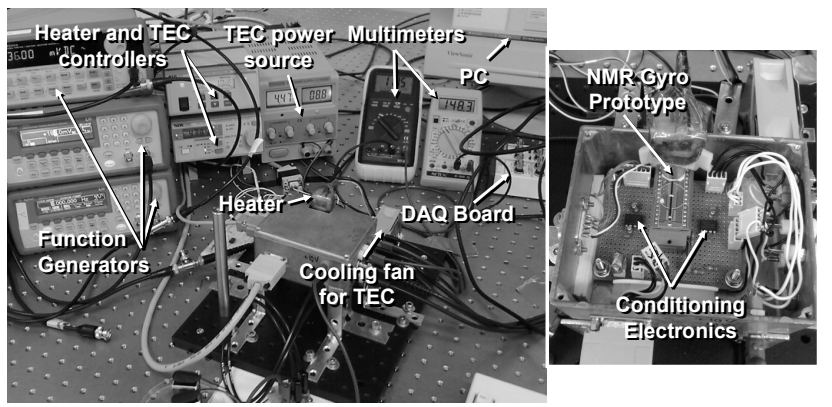

Figure 2: Experimental setup for thermal characterization of the NMR Gyroscope.

\section{Temperature Measurements}

Two temperature sensors (Thorlabs TH100PT) were placed inside of the device, but outside of the cell (Fig. 1). The first sensor provided temperature measurements a few millimeters above the cell. The second sensor provided measurements below the base plate and near the VCSEL. Two additional temperature sensors (Thorlabs TH10K) were used to measure the heater and TEC temperatures. In addition to these four direct temperature measurements, two indirect temperature measurements, the VCSEL and cell temperatures, were obtained by sweeping the VCSEL current and measuring the amount of light intensity absorbed by the $\mathrm{Rb}$ in the cell. The emitted laser wavelength has a temperature and current dependence according to:

$$
\lambda=\lambda_{0}+\alpha \cdot\left(I-I_{0}\right)+\beta \cdot\left(T-T_{0}\right),
$$

where $\quad \lambda_{0}=795 \mathrm{~nm}, \quad \alpha=0.3 \mathrm{~nm} / \mathrm{mA}, \quad \beta=0.055 \mathrm{~nm} /{ }^{\circ} \mathrm{C}$, $\mathrm{I}_{0}=4.4 \mathrm{~mA}$ and $\mathrm{T}_{0}=25^{\circ} \mathrm{C}$. The parameters $\alpha$ and $\beta$ describe how much the laser wavelength deviates from $\lambda_{0}$ due to changes in the VCSEL current or temperature.

As the resonance peaks occur when the VCSEL's wavelength equals the energy level of the ${ }^{87} \mathrm{Rb} \mathrm{D}_{1}$ transition $(\lambda=795 \mathrm{~nm})$, the VCSEL's temperature can be obtained by sweeping its current and using Equation (1). The sweep period was 5 seconds, and therefore in the presence of an absorption peak, the VCSEL temperature can be indirectly measured every 5 seconds.

In addition to the VCSEL temperature the cell temperature can also be obtained by observing the absorption peak linewidth. If light with intensity $\mathrm{J}_{0}$ is transmitted through a cell with path length $\mathrm{L}$, the output light intensity can be approximated by [7]:

$$
J=J_{0} \cdot e^{-N \sigma L},
$$

where $\mathrm{N}$ is the number density of the alkali vapor and $\sigma$ is the absorption cross-section, which has a Lorentzian frequency distribution according to:

$$
\sigma=c \cdot f_{\text {osc }} \cdot r_{e} \cdot \frac{\Gamma / 2}{\left(f_{0}-f\right)^{2}+(\Gamma / 2)^{2}},
$$

where $c$ is the speed of light, $f_{o s c}=0.342$ is the oscillator strength for the $D_{1}$ transition of ${ }^{87} R b, r_{e}$ is the electron radius, $\Gamma$ is the linewidth of the broadening, $f_{0}$ is the absorption frequency and $\mathrm{f}$ is the laser light frequency. The rubidium number density can be written as $\mathrm{N}=\mathrm{P} /\left(\mathrm{k}_{\mathrm{B}} \cdot \mathrm{T}\right)$, where $k_{B}$ is the Boltzmann constant, $T$ is the $R b$ vapor temperature and $\mathrm{P}$ is the $\mathrm{Rb}$ vapor pressure. The $\mathrm{Rb}$ vapor pressure can be obtained in Torr from [8]:

$$
\log _{10} P_{R b}=15.88253-\frac{4529.635}{T}+0.00058663 \cdot T-2.99138 \cdot \log _{10} T
$$

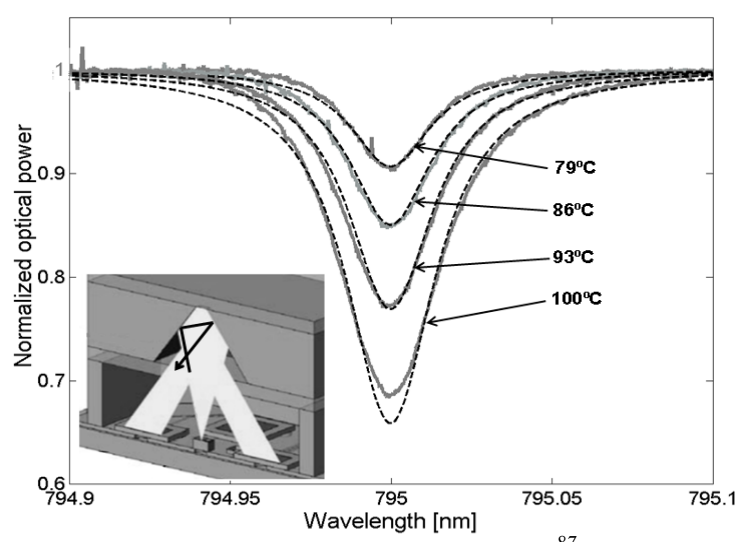

Figure 3: Normalized wavelength spectrum of ${ }^{87} \mathrm{Rb} \mathrm{D}_{1}$ transition at different temperatures (solid) compared to pressure broadened absorption model (dashed).

Fig. 3 shows a comparison between the described pressure broadened absorption model and the normalized ${ }^{87} \mathrm{Rb} \mathrm{D}_{1}$ line absorption profile measured at different temperatures. The insert in Fig. 3 shows the laser pathway inside the cell [9] ( $\mathrm{L}=2.15 \mathrm{~mm})$.

To be able to accurately reproduce the experimental conditions in the FE model, the device was first operated in a steady-state and the initial temperatures were recorded. All measurements were taken at a sampling frequency of $100 \mathrm{~Hz}$ with the TEC operating at a constant current of $2 \mathrm{~A}$, yielding a TEC temperature of $-30^{\circ} \mathrm{C}$.

Two different sets of measurements were collected under slightly different conditions. In the first case, measurements were taken when the heater cooled down from $195^{\circ} \mathrm{C}$ to $185^{\circ} \mathrm{C}$. In the second set of experiments, the heater temperature was increased from $180^{\circ} \mathrm{C}$ to $205^{\circ} \mathrm{C}$.

\section{Measurements Filtering}

The filter used in the direct experimental measurements is described in [10] and is widely used in experiments involving multi-exponential decays with time-constants spanning several orders of magnitude (for example, MEMS thermal responses). In such cases, uniform 
sampling of thermal transients delivers an oversampling of the steady state. To solve this problem and reduce the noise level, a logarithmic time-scale transformation is applied to these transients. In the experiments under consideration, the filtered transients are chosen to have 40 points per decade resulting in a total of 191 and 197 samples for the first and second set of measurements, respectively.

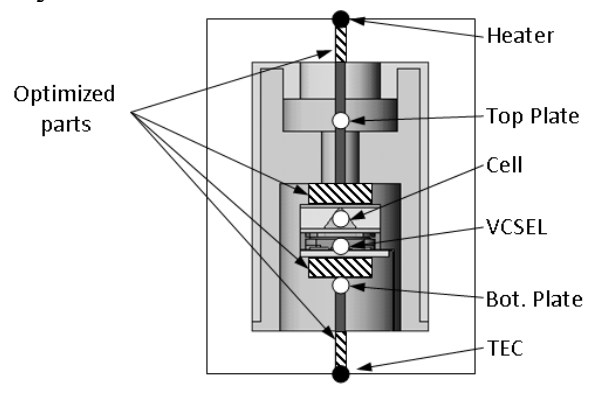

Figure 4: Cross section of NMR Gyroscope showing controlled nodes for deriving the thermal model and optimized parts.

Table 1: Material Properties*.

\begin{tabular}{|c|c|c|c|}
\hline Material & $\begin{array}{c}\text { Thermal } \\
\text { Conductivity } \\
{[\mathbf{W} /(\mathbf{m} \cdot \mathbf{K})]}\end{array}$ & $\begin{array}{c}\text { Specific Heat } \\
\text { Capacity } \\
{[\mathbf{J} /(\mathbf{k g} \cdot \mathbf{K})]}\end{array}$ & $\begin{array}{c}\text { Density } \\
{\left[\mathbf{k g} / \mathbf{m}^{3}\right]}\end{array}$ \\
\cline { 1 - 4 } Silicon & 163 & 703 & 2330 \\
Air & 0.026 & 1100 & 11.7 \\
Polyimide & 0.2 & 1100 & 1300 \\
HD4110 & 2 & 1500 & 1300 \\
Loctite 1C-LV & 1.2 & 730 & 2200 \\
Pyrex 7440 & 400 & 385 & 8700 \\
Copper & 0.5 & 1100 & 1100 \\
Amethyst SL &
\end{tabular}

*Air has temperature dependent properties, shown values correspond to $25^{\circ} \mathrm{C}$.

\section{THERMAL MODEL}

An FE model that reproduces the real prototype geometry was developed using COMSOL $\AA$. In order to reduce the complexity of the FE model, the external heater, the TEC, and the internal temperature sensors were omitted from the model. The heating and cooling copper wires were limited to $2 \mathrm{~mm}$ in length, as shown in Fig. 4. The heater and TEC temperatures obtained with the experimental setup were applied as loads in the FE model (black dots in Fig. 4). The material properties that were used are shown in Table 1.

\section{Optimization}

Since a simplified FE model was used the thermal conductivities of some parts of the device needed to be optimized in order to match the simulation results with the measurements. The four parts chosen are shown in Fig. 4, including the top portion of the heating wire, both copper plates, and the bottom portion of the cooling wire. The optimization process [11] gives us the four thermal conductivities (see Table 2) which minimize the steady- state temperature errors between the FE model and measurements (see Table 3).

Table 2: Optimized Thermal Conductivities

\begin{tabular}{|c|c|}
\hline Location & $\begin{array}{c}\text { Thermal } \\
\text { Conductivity } \\
{[\mathbf{W} /(\mathbf{m} \cdot \mathbf{K})]}\end{array}$ \\
\hline Top Portion of heating wire & 40.4 \\
Top Copper Plate & 5.5 \\
Bottom Copper Plate & 401 \\
Bottom Portion of cooling wire & 21.8 \\
\hline
\end{tabular}

Table 3: Steady State Optimization Results.

\begin{tabular}{|c|c|c|c|c|c|c|}
\cline { 2 - 7 } \multicolumn{1}{c|}{} & \multicolumn{3}{c|}{ Set 1 } & \multicolumn{3}{c|}{ Set 2 } \\
\cline { 2 - 7 } \multicolumn{1}{c|}{} & Simul. & Meas. & Error & Simul. & Meas. & Error \\
\hline Top Plate & 118.8 & 117.8 & 1.0 & 130.8 & 130.9 & -0.1 \\
Cell & 91.0 & 92.0 & -1.0 & 100.9 & 98 & 2.9 \\
VCSEL & 53.2 & 53.0 & 0.2 & 60.6 & 59.5 & 1.1 \\
Bot. Plate & 50.1 & 53.7 & -3.6 & 57.3 & 59.6 & -2.3 \\
\hline
\end{tabular}

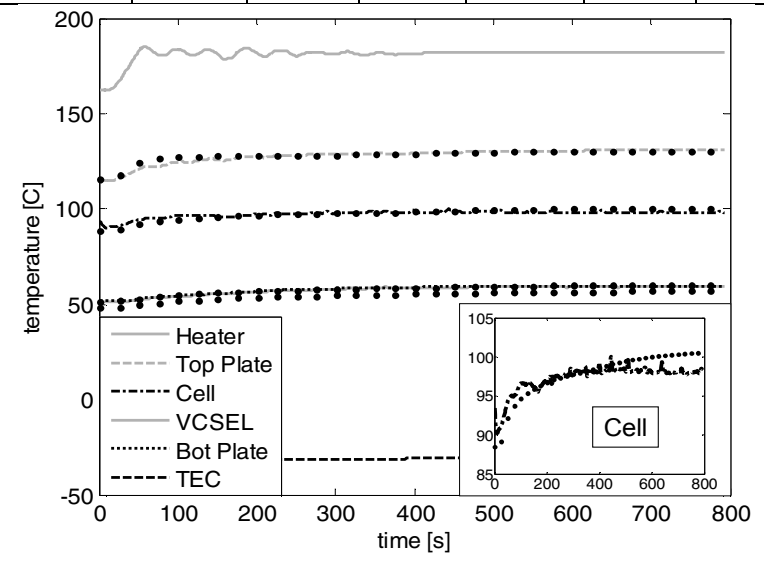

Figure 5: Illustration of matching between direct experimental measurements (lines) and optimized FE model (dotted lines) at chosen locations of the NMR Gyroscope.

Table 4: Errors comparing FE model and measurements.

\begin{tabular}{|c|c|c|c|c|c|c|}
\hline \multirow{2}{*}{ Location } & \multicolumn{2}{|c|}{$\begin{array}{c}\text { RMS error } \\
{\left[{ }^{\mathbf{}} \mathbf{C}\right]}\end{array}$} & \multicolumn{2}{c|}{$\begin{array}{c}\text { ABS error } \\
{\left[{ }^{\mathbf{}} \mathbf{C}\right]}\end{array}$} & \multicolumn{2}{|c|}{$\begin{array}{c}\text { Steady-State } \\
\text { error } \\
{\left[{ }^{\mathbf{}} \mathbf{C}\right]}\end{array}$} \\
\cline { 2 - 7 } & set 1 & set 2 & set 1 & set 2 & set 1 & set 2 \\
\hline Top Plate & 1.3 & 1.3 & 1.6 & 4.3 & 1.2 & 0.4 \\
Cell & 0.7 & 1.5 & 1.7 & 5.0 & 0.8 & 2.4 \\
VCSEL & 0.5 & 0.5 & 1.4 & 1.2 & 0.5 & 0.8 \\
Bot. Plate & 3.5 & 3.2 & 4.2 & 3.8 & 3.3 & 2.7 \\
\hline
\end{tabular}

A comparison between the thermal transients of the FE model and the experimental results for the second measurement set is shown in Fig. 5, with a close-up view of the cell in the inset picture. A summary of the errors for both measurement sets is given in Table 4. Note that the heater and TEC are not included in Table 4, since their errors are zero (were used as inputs in the FE model).

\section{COMPACT MODEL}

Once the FE model has been optimized to match the experimental measurements, the next step is to build a 
CTM from the FE model. A CTM is a reduced order model which replicates with high accuracy the thermal behavior of the FE model, and in turn the physical device. The methodology is based on a Sequential Forward Selection (SFS) process described in [12]. The CTM is represented as an $\mathrm{RC}$ network using a thermo-electrical analogy. For the presented device, the resulting CTM has a total of 12 nodes.

Table 5 shows a summary of RMS, absolute, and steady-state errors when comparing the FE model step response transients to the obtained CTM results. The large absolute errors correspond to the central portion of the step response transients. The steady-state errors are below $0.1^{\circ} \mathrm{C}$ and the RMS errors are approximately $0.15^{\circ} \mathrm{C}$ for all four transients.

Table 5: Errors comparing CTM and FE model.

\begin{tabular}{|c|c|c|c|}
\hline Location & $\begin{array}{c}\text { RMS error } \\
{\left[{ }^{\mathbf{}} \mathbf{C}\right]}\end{array}$ & $\begin{array}{c}\text { ABS error } \\
{\left[{ }^{\mathbf{o}} \mathbf{C}\right]}\end{array}$ & $\begin{array}{c}\text { Steady-State } \\
\text { error } \\
{\left[{ }^{\mathbf{}} \mathbf{C}\right]}\end{array}$ \\
\hline Top Plate & 0.15 & 0.62 & -0.008 \\
Cell & 0.17 & 0.75 & 0.006 \\
VCSEL & 0.14 & 0.39 & 0.021 \\
Bot. Plate & 0.13 & 0.36 & 0.051 \\
\hline
\end{tabular}

\section{DISCUSSION}

The ultimate step of the presented process is comparison of the CTM with the experimental measurements (Table 6). This comparison highlights that the largest contribution to the CTM error is the FE model optimization step. The FE model currently does not match the experimental measurements with sufficient accuracy. Better control of the boundary conditions by utilizing an improved experimental setup needs to be achieved to reduce the uncertainties of the FE model. In addition, an accurate FE model that reproduces more details of the physical device can be implemented to reduce these errors.

Table 6: Errors comparing CTM model and measurements.

\begin{tabular}{|c|c|c|c|c|c|c|}
\hline \multirow{2}{*}{ Location } & \multicolumn{2}{|c|}{$\begin{array}{c}\text { RMS error } \\
{\left[{ }^{\mathbf{}} \mathbf{C}\right]}\end{array}$} & \multicolumn{2}{c|}{$\begin{array}{c}\text { ABS error } \\
{\left[{ }^{\mathbf{}} \mathbf{C}\right]}\end{array}$} & \multicolumn{2}{|c|}{$\begin{array}{c}\text { Steady-State } \\
\text { error } \\
{\left[{ }^{\mathbf{C}} \mathbf{C}\right]}\end{array}$} \\
\cline { 2 - 7 } & set 1 & set 2 & set 1 & set 2 & set 1 & set 2 \\
\hline Top Plate & 1.0 & 1.0 & 1.6 & 4.2 & 1.2 & 0.4 \\
Cell & 0.6 & 1.7 & 2.0 & 5.0 & 0.7 & 2.3 \\
VCSEL & 0.8 & 0.6 & 2.0 & 1.3 & 0.9 & 0.6 \\
Bot. Plate & 2.6 & 3.8 & 3.8 & 4.6 & 3.0 & 2.9 \\
\hline
\end{tabular}

The presented results demonstrate that the methodology developed in this work can be used to indirectly obtain temperature estimates at critical locations of the device where direct measurements are not possible. Accurate predictive models are needed for a broad class of atomic MEMS, where temperature at unobservable locations of the system needs to be controlled with high accuracy $(<1 \mathrm{mK})$.

\section{ACKNOWLEDGEMENTS}

The authors thank Dr. Max Perez, and Monty Rivers for their contributions to this work. One of the authors (M.S.) is enjoying a Balsells Fellowship. This work was partially supported by NIST, contract 70NANB5H1235.

\section{REFERENCES}

[1] E. Hodby, E.A. Donley and J. Kitching, "Differential atomic magnetometry based on a diverging laser beam", Appl. Phys. Lett., vol. 91, pp. 011109, 2007.

[2] E.J. Eklund, M.C. Rivers and A.M. Shkel, "Folded Micro-Gyroscope with Nuclear Magnetic Resonance Sensing”, Patent pending, UC Case No. 2008-125.

[3] E.J. Eklund, "Microgyroscope Based on SpinPolarized Nuclei", Ph.D. dissertation, University of California at Irvine, Irvine, CA, USA, 2008.

[4] K.F. Woodman, P.W. Franks and M.D. Richards, "The Nuclear Magnetic Resonance Gyroscope: a Review," Journal of Navigation, vol. 40(3), pp. 366-384, 1987.

[5] A.T. Nicol, "Nuclear Moment Alignment, Relaxation and Detection Mechanisms", Guidance and Control Systems Div., Litton Systems Inc., Tech. Rep. ADA 115203, February, 1982.

[6] S. Appelt, T. Unlu, K. Zilles, N. J. Shah, S. BaerLang, and H. Halling, "Experimental studies of rubidium absolute polarization at high temperatures," Appl. Phys. Lett., vol. 75(3), pp. 427-429, 1999.

[7] A.C.G. Mitchell and M.W. Zemansky, "Resonance Radiation and Excited Atoms," Cambridge University Press, 1961.

[8] D.A. Steck, "Rubidium 87 D line data", Theoretical Division (T-8), Los Alamos National Laboratory, Tech. Rep. MS B285, 2001.

[9] M.A. Perez, U. Nguyen, S. Knappe, E. Donley, J. Kitching and A.M. Shkel, "Rubidium Vapor Cell with Integrated Nonmetallic Multilayer Reflectors," in Conf. Proc. MEMS, Tucson, January 13-17, 2008, pp. 790-793.

[10]J. Palacín, S. Marco, J. Samitier, "Suboptimal Filtering and Nonlinear Time Scale Transformation for the Analysis of Multiexponential Decays," IEEE Trans. Instrum. Meas., vol. 50, pp. 135-140, 2001.

[11]Coleman, T.F. and Y. Li, "An Interior, Trust Region Approach for Nonlinear Minimization Subject to Bounds," SIAM Journal on Optimization, vol. 6, pp. 418-445, 1996.

[12]M. Salleras, et al., "A methodology to extract dynamic compact thermal models under time-varying boundary conditions: Application to a thermopile based IR sensor," Microsystem Technologies, vol. 12, pp. 2129, 2005.

\section{CONTACT}

* M. Salleras, tel: +1-949-824-4901; msallera@uci.edu 